This item was submitted to Loughborough's Research Repository by the author.

Items in Figshare are protected by copyright, with all rights reserved, unless otherwise indicated.

\title{
The role of contextual information during skilled anticipation
}

PLEASE CITE THE PUBLISHED VERSION

https://doi.org/10.1177/1747021817739201

PUBLISHER

SAGE Publications ( Experimental Psychology Society

VERSION

AM (Accepted Manuscript)

PUBLISHER STATEMENT

This work is made available according to the conditions of the Creative Commons Attribution-NonCommercialNoDerivatives 4.0 International (CC BY-NC-ND 4.0) licence. Full details of this licence are available at: https://creativecommons.org/licenses/by-nc-nd/4.0/

\section{LICENCE}

CC BY-NC-ND 4.0

\section{REPOSITORY RECORD}

Murphy, Colm P., Robin Jackson, and A. Mark Williams. 2019. "The Role of Contextual Information During Skilled Anticipation". figshare. https://hdl.handle.net/2134/26770. 


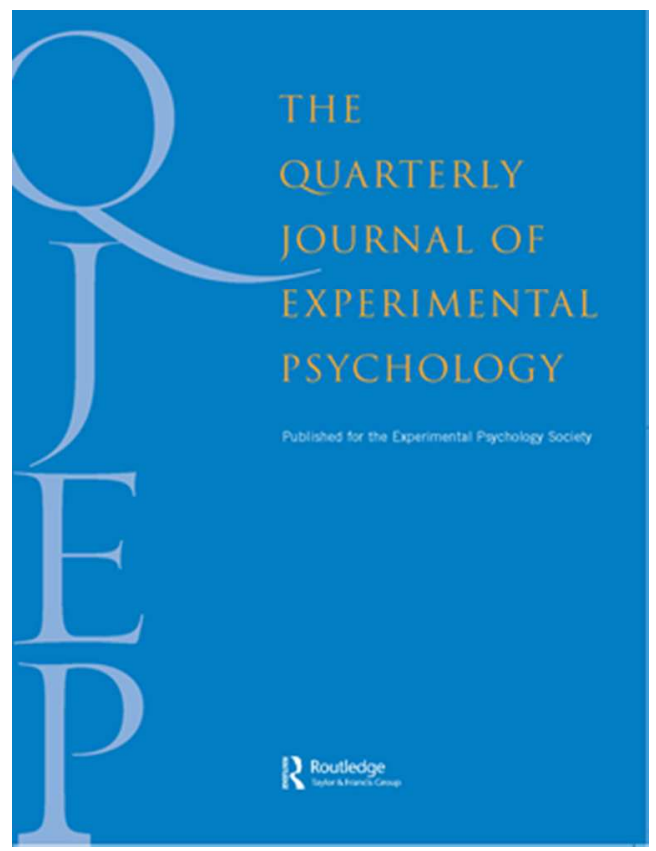

\section{The Role of Contextual Information during Skilled Anticipation}

\begin{tabular}{|r|l|}
\hline Journal: & Quarterly Journal of Experimental Psychology \\
\hline Manuscript ID & QJE-STD 16-360.R2 \\
\hline Manuscript Type: & Standard Article \\
\hline Date Submitted by the Author: & n/a \\
\hline Complete List of Authors: & $\begin{array}{l}\text { Murphy, Colm; Brunel University London, Department of Life Sciences, ; St } \\
\text { Mary's University, Expert Performance and Skill Acquisition Research } \\
\text { Group, School of Sport, Health and Applied Science, } \\
\text { Jackson, Robin; Loughborough University, School of Sport, Exercise and } \\
\text { Health Sciences, } \\
\text { Williams, A.; University of Utah, Department of Health, Kinesiology and } \\
\text { Recreation }\end{array}$ \\
\hline Keywords: & expertise, perceptual-cognitive skill, context, awareness, cognition \\
\hline &
\end{tabular}




\author{
Author Note \\ None of the authors have any links to companies or manufacturers who would benefit from \\ this work. There are no conflicts of interest present.
}

Date of Submission: October 16, 2016 


\begin{abstract}
In dynamic, temporally-constrained tasks, individuals often need to anticipate what will happen next prior to information becoming available within the environment. In such situations, the availability of contextual information can facilitate anticipation, often in conjunction with postural information. While many researchers have identified the specific sources of postural information facilitating anticipation, few have investigated the specific sources of contextual information employed. In two experiments, we presented skilled and less-skilled tennis players with animations of rallies from real matches that omitted access to postural information from the opponent, constraining participants to anticipate based on contextual information alone. In Experiment 1, participants anticipated the outcome of an opponent's shot under three conditions in which the sequence length (i.e., number of shots in a rally) preceding the same occluded shot was varied. Participants anticipated shot direction more accurately when the preceding shot sequence was presented than not. In Experiment 2, we presented animations that depicted the ball, the players, or both, in either dynamic or still form. Those conditions in which only the ball was depicted yielded the lowest response accuracy scores. It appears that information from the player and ball motion is required to provide the context under which skilled performers can consciously pick up and utilise information to anticipate more accurately than their less-skilled counterparts.
\end{abstract}

Keywords: expertise; perceptual-cognitive skill; context; awareness; cognition. 


\section{The Role of Contextual Information during Skilled Anticipation}

At the highest levels of performance in dynamic, temporally-constrained domains, such as military combat, sport, and law enforcement, performers often have to make quick and accurate judgments based on minimal information. In such situations skilled performers extract and utilise postural information to anticipate the opponent's intentions more effectively than their less-skilled counterparts (e.g., Abernethy \& Russell, 1987; Williams \& Burwitz, 1993). Moreover, researchers have identified the specific sources of postural information employed, which is task specific, such as, for example, the hips in the soccer penalty kick (Causer, Smeeton, \& Williams, 2017) or the arm and racket in the tennis serve (Jackson \& Mogan, 2007). In fast ball sports, the time taken to process and respond to ball flight information often exceeds the time it takes for the ball to pass the receiving player (Singer, 2000; Williams, Davids, \& Williams, 1999), and under particularly extreme time constraints, the same may even be true when responding based on pertinent postural cues picked up some brief moments prior to event occurrence (Triolet, Benguigui, Le Runigo, \& Williams, 2013). When skilled performers are not afforded the time to wait for pertinent postural cues to become available, they are thought to rely on contextual information to anticipate the opponent's intentions (Abernethy, Gill, Parks, \& Packer, 2001; Müller \& Abernethy, 2012). In this paper, we focus on determining the extent to which performers can use different sources of contextual information (i.e., shot sequencing, ball and player motion and positioning) to anticipate independent of postural information.

Buckolz, Prapavesis and Fairs (1988) suggested that two types of information can be used to anticipate the intentions of an opponent, namely, postural and contextual information. Postural information arises from the movement pattern of the opponent, whereas contextual information is available prior to pertinent postural cues becoming available. Moreover, contextual information remains available as the movement pattern of the opponent develops. 
Buckolz et al. suggested that contextual information exists in numerous forms such as knowledge of the opponent's strengths and weaknesses, climatic conditions, and the relative positioning of the players on the playing terrain. In recent years, researchers have begun to investigate how certain sources of contextual information such as score-dependent patterns of play (Farrow \& Reid, 2012), knowledge of an opponent's action tendencies (Navia, van der Kamp, \& Ruiz, 2013), or court positioning of the opponent (Loffing \& Hagemann, 2014) influence anticipation judgments in skilled performers. It appears that skilled performers are very adept at using this information to adjust their expectancies relating to upcoming events.

It is thought that as a result of their extended experience within a domain skilled performers develop Long Term Working Memory (LTWM) skills (Ericsson \& Kintsch, 1995), which allow rapid access to, and retrieval of, information stored in Long Term Memory (LTM). Skilled performers can encode the presented information and associate it with a retrieval cue in Short Term Memory (STM), which allows access to information in LTM about the relationship between the presented information and potential event outcomes. These retrieval structures allow skilled performers access to task-relevant options, which can be evaluated to inform an accurate judgment rather than merely prescribing a set response (Ericsson, Patel, \& Kintsch, 2000). Skilled performers are thought to adjust their expectancies of potential event outcomes based on contextual information available in addition to emerging postural cues, the suggestion being that their more advanced domain-specific knowledge facilitates integration of pertinent contextual information during the anticipation process (e.g., see Loffing \& Hagemann, 2014).

Several researchers have demonstrated that contextual information influences anticipation when presented in addition to postural cues (e.g., Loffing \& Hagemann, 2014; McRobert, Ward, Eccles, \& Williams, 2011). However, contextual information may be of particular importance for anticipating effectively when pertinent postural cues are not 
available for processing. Skilled performers appear able to make remarkably accurate anticipation judgments in advance of pertinent postural cues becoming available. For example, skilled racket sports players have been shown to accurately anticipate the outcome of an opponent's shot approximately $580 \mathrm{~ms}$ and $720 \mathrm{~ms}$ prior to the opponent striking the ball in squash (Abernethy et al., 2001) and tennis (Triolet et al., 2013), respectively. Abernethy et al. (2001) suggest that these early occurring anticipatory movements are likely to be due to the use of contextual information picked up from sources such as the preceding shot sequence. Triolet et al. (2013) further observed that highly skilled tennis players often began to respond to the opponent's shot early because they were placed under extreme time constraints, for example, in situations when the opponent was attacking from inside the court.

Murphy et al. (2016) sought to clarify whether contextual information, which may normally be relied upon for anticipation far in advance of a critical event, can facilitate anticipation independent of postural information. As well as viewing video footage of rallies from real tennis matches, skilled and less-skilled tennis players viewed animations of the same rallies in which each of the players were replaced by a cylinder and their rackets were not visible. When viewing the animations, participants were constrained to anticipate based on contextual information alone, eliminating the possibility that their judgments could be based on very early occurring postural cues. The aim in using this approach was to determine, with a high level of experimental control, whether contextual information can be used to anticipate effectively, independent of pertinent postural cues. Although participants anticipated more accurately when viewing video compared with animated footage, both groups were more accurate than chance when viewing the animations, with the skilled participants being most accurate. It appears that skilled performers can rely solely on contextual information to anticipate highly accurately; the suggestion being even under such 
conditions skilled participants are able to access associated task-relevant information from LTM to facilitate more accurate anticipation when compared with less-skilled participants.

Skilled racket sports players have been shown to play specific shots in response to certain shot sequences (McGarry \& Franks, 1996). In each of the research studies conducted by Abernethy et al. (2001), Triolet et al. (2013), and Murphy et al. (2016), participants had access to contextual information that could, potentially, be picked up from the shot sequence preceding the to-be-anticipated shot, with the sequence length varying from trial to trial. This approach is in stark contrast to most research on anticipation to date in which participants have either been presented with isolated actions such that information about the preceding sequence of events is unavailable (e.g., see Jackson \& Mogan, 2007; Müller, Abernethy, Eid, McBean, \& Rose, 2010) or alternatively, with sequences that have been presented for a fixed period of time or number of events (e.g., see Roca, Ford, McRobert, \& Williams, 2011; Ryu, Abernethy, Mann, Poolton, \& Gorman, 2013). In such experimental set-ups, the influence that the preceding sequence of events may have on anticipation is controlled or ignored. Only a few researchers have attempted to determine how having access to the preceding sequence of events influences performance (e.g., see Gray, 2002a, 2002b; Loffing, Stern, \& Hageman, 2015).

Gray (2002a, 2002b) used a simulated baseball batting task to demonstrate that the expectancies of college level baseball batters are influenced by the preceding sequence of pitches. When three fast pitches were followed by a fast pitch, their batting was more accurate than if the three fast pitches were followed by a slow pitch. Loffing, Stern, and Hagemann (2015) presented skilled and novice volleyball players with sequences of four attacking shots which had been manipulated to either always present smashes, lobs, or an alternating pattern of the two shots prior to anticipating a shot which was either congruent or incongruent with the preceding sequential pattern of shots played. Their findings suggest that 
expectations of action outcomes were more strongly affected by these sequences in skilled compared with less-skilled participants.

It has been reported that skilled performers are better than less-skilled performers at picking up repeated patterns in an opponent's game to facilitate anticipation (Farrow \& Reid, 2012; Milazzo, Farrow, Ruffault, \& Fournier, 2015). McRobert et al. (2011) presented skilled and less-skilled cricket batters with videos of six "fast" and "slow" bowlers presented in a random order or videos of four "fast" bowlers presented in blocks, such that they viewed each of the four bowlers for six bowls in a row. Performance levels were higher when viewing the same bowler over repeated trials. The skilled batters adapted their gaze behaviour when repeatedly viewing the same bowler. The authors suggested that the contextual information provided by the preceding actions of the bowler allowed the skilled performers to adapt their gaze behaviour to pick up information from relevant locations more efficiently than when this information was not available. While this body of work suggests that the sequence of events preceding a critical event (e.g., hand-ball release, racket-ball contact of an opponent) influences anticipation, no published research has to our knowledge investigated whether the strength of this influence is affected by the number of events in the preceding sequence.

A common approach taken by researchers attempting to identify the specific sources of information that facilitate anticipation is to manipulate the experimental task (Williams \& Ericsson, 2005). Several researchers have employed the spatial occlusion paradigm by artificially removing potential cue sources from the visual display such that their relevance as visual cues can be assessed using video simulations (e.g., Abernethy, 1990; Jackson \& Mogan, 2007; Williams \& Davids, 1998) and point-light or stick figure displays (e.g., Abernethy \& Zawi, 2007; Abernethy, Jackson, \& Zawi, 2008; Huys et al., 2009). If there is a significant deterioration in accuracy when one potential source of information is occluded, this element is assumed to be an important cue for anticipation (e.g., the arm and racket in 
racket sports, Abernethy \& Zawi, 2007; Abernethy et al., 2008; Shim, Carlton, \& Kwon, 2006).

Similarly, some researchers have manipulated the information they present to participants to determine the sources of information facilitating expert pattern recognition and recall (e.g., Gorman, Abernethy \& Farrow, 2011; North, Ward, Ericsson, \& Williams, 2011; Williams, North, \& Hope, 2012). In two experiments, Williams et al. (2012) manipulated videos of sequences from soccer matches such that they were presented in either still or dynamic format, and then presented videos in which central or peripheral elements were omitted from the dynamic display. They reported a decrement in pattern recognition for skilled players in the still relative to the dynamic condition (see also Sebanz and Shiffrar, 2009) and subsequently, when central elements were omitted from the display. Williams et al. (2012) suggest a key mechanism underpinning skilled pattern recognition is the effective extraction of motion information and that only the relative motions of a few key features (e.g., central midfielders and offensive players) is necessary for effective recognition of domain-specific patterns. However, some contradictory findings have been reported using pattern recall and decision-making tasks (Gorman et al., 2011; Gorman, Abernethy, \& Farrow, 2013). For example, Gorman et al. (2013) reported that pattern recall error was lower and decision making accuracy higher when viewing a still compared to a dynamic display involving basketball plays. This body of research is pertinent because it has been suggested that the ability to recognise and/or recall domain-specific patterns may act as an important precursor to effective anticipation (Cañal-Bruland \& Williams, 2010; Gorman, Abernethy, \& Farrow, 2012). What is particularly clear, however, is that the manipulation of representative test stimuli, is a useful way to determine the sources of information used by performers to make accurate judgments, which could ultimately lead to the identification of underlying mechanisms. 
To date, those attempting to identify the sources of contextual information that contribute to skilled anticipation (e.g., Loffing \& Hagemann, 2014; McRobert et al., 2011) have done so with an emphasis on determining how this information interacts with postural cues. However, when placed under extreme time constraints, the time it takes for the action to be carried out may be so minimal that waiting for pertinent postural cues to become available is not feasible (Triolet et al., 2013). In such situations performers would be constrained to rely on contextual information alone to anticipate effectively. In this paper, we provide a novel contribution by determining the extent to which specific sources of contextual information facilitate accurate anticipation independent of pertinent postural cues.

\section{Experiment 1}

Crognier and Féry (2005) examined whether tennis players anticipate more effectively in situations in which they can impose their game on the opponent compared to situations in which they have fewer possibilities to do so. In order to examine this issue, experienced tennis players played points against an opponent in one of three conditions involving increasing levels of tactical initiative. The experiment was set up in such a way that sequence length was shortest in the low tactical initiative condition and longest in the high tactical initiative condition. Although the participants anticipated the direction of the opponent's shot most accurately following the longest sequences, it is impossible to disentangle the relative importance of tactical initiative and the impact of rally length.

In Experiment 1, we examined whether contextual information can be picked up from the sequence of shots played prior to a critical event (i.e., racket-ball contact of an opponent in this study) to facilitate anticipation. A secondary aim focused on determining whether the ability to use this information develops with increasing skill level. Finally, we investigated whether the length of the preceding sequence affects anticipation. We presented skilled and 
less-skilled tennis players with animations of sequences of shots played by players in real matches. In these animations, the bodies of the players were replaced by a cylinder and rackets were not visible (see Murphy et al., 2016), such that the observer was constrained to anticipate based on contextual information alone. To determine whether the preceding shot sequence provides contextual information which can be used to facilitate anticipation, we compared the ability of skilled and less-skilled participants to anticipate the intentions of an opponent when viewing trials that presented one, three or five shots prior to the same shot played by the opponent, occluded at racket-ball contact.

First, we hypothesised, based on the findings of Murphy et al. (2016), that for both groups, accuracy would be significantly higher than chance. Second, based on the same research, we expected skilled participants to be more accurate than less-skilled participants. Third, to our knowledge, the only research that has provided any indication as to whether the sequence length preceding a critical event affects anticipation is that conducted by Crognier and Féry (2005), albeit as mentioned above a confound existed in the design. This aspect of the experiment was therefore exploratory. Fourth, based on the findings of several previous research studies demonstrating that the judgments of skilled performers are affected by the presentation of contextual information over and beyond the presentation of postural information (e.g., Crognier \& Féry, 2005; Farrow \& Reid, 2012; Loffing \& Hagemann, 2014), we hypothesised that skilled participants would anticipate more accurately when presented with the preceding shot sequence (i.e., three or five shot conditions) in addition to viewing the final occluded shot. We expected that the superior LTWM skills (Ericsson \& Kintsch, 1995) possessed by the skilled participants would allow them to access potentially relevant alternatives associated with the presented contextual information, allowing them to adjust their expectancies to make more accurate judgments than when this additional 
information was not presented. Conversely, we expected the accuracy of the less-skilled participants to be unaffected by the presentation of the preceding shot sequence.

\section{Method}

\section{Participants}

Altogether, 12 skilled $\left(M_{\mathrm{age}}=27.1, S D=4.7\right)$ and 12 less-skilled $\left(M_{\mathrm{age}}=24.7, \mathrm{SD}=5.3\right)$ male tennis players participated. Skilled and less-skilled participants had a mean of 20.2 (SD $=4.6)$ and $4.3(S D=3.6)$ years of tennis playing experience, respectively. Skilled participants held British Tennis ratings of 1.1 to 4.1 , whereas less-skilled participants held ratings of 10.2 or did not hold a rating. British Tennis ratings range from 1.1 (highest) to 10.2 (lowest). Skilled participants reported having played a mean of $54.1(S D=24.1)$ matches per year, whereas less skilled players did not play competitively. One participant in the less-skilled group was left-handed and the rest were right-handed players. Participants had normal or corrected vision. Those with corrected vision wore glasses or contact lenses while participating. The research was carried out in line with the lead university's research ethics guidelines. Participants provided informed consent prior to taking part and knew that they could withdraw from testing at any time without consequence.

\section{Test Stimuli}

Player movement and ball trajectory data (Hawk-Eye Innovations Ltd., Basingstoke, UK) from professional tennis matches played at the AEGON Championships (2013) were used to create the test stimuli. The data were input into a rendering engine (Julien Pansiot, London, UK) to generate animations of rallies which could then be viewed on VLC media player (VideoLAN, Paris, France). The test stimuli were animations of real tennis rallies which displayed two players moving around the court playing a point, but which omitted the 
players' bodies and rackets such that they were depicted as a blue and a red cylinder and their rackets were not visible, while the ball was depicted as a yellow dot (see Figure 1). Pinnacle Studio 15 editing software (Pinnacle, Ottawa, Canada) was used to edit the animations to occlude at the opponent's racket-ball contact.

$<<$ Insert Figure 1 near here $>>$

The criteria used for selecting the shot on which to occlude the footage was the same as that used by Murphy et al. (2016). Shots in which the receiving player was placed under extreme time constraints were selected from the database of rallies. In such conditions (e.g., when one player is attacking the other from inside the court or the distance between the two players is smaller than usual), players are constrained to respond earlier than usual to get to, and return, the opponent's shot effectively (Triolet et al., 2013). Furthermore, a minimum of six shots needed to be exchanged between the two players in the rally for it to be suitable for creating the longest sequence length condition (five preceding shots plus the final occluded shot). A total of 44 sequences of six shots were selected based on these criteria.

In an effort to ensure that we only used trials in which the shot sequence preceding the final occluded shot presented relevant contextual information, three experienced coaches independently viewed each of the 44 animated sequences that were occluded at the opponent's racket-ball contact on the sixth shot. All coaches had over 10 years of experience and had coached players of National and/or International level as well as having played at that level. For each of the 44 trials, each coach rated the extent to which the preceding shot sequence would contribute to successfully anticipating the outcome of the final occluded shot on a scale of 1 to 5 with 1 being "not at all" and 5 "extremely". Only sequences in which coaches reported an average rating greater than or equal to 4 were used as test stimuli, yielding a total of 23 trials. A similar procedure has been used in pattern recall and 
recognition research, where experienced coaches have identified structure in sequences of play to ensure that the footage being used is representative of structured gameplay (Gorman et al., 2012; North, Williams, Hodges, Ward, \& Ericsson, 2009).

To determine the effect that increasing the number of shots in the sequence preceding the final occluded shot had on anticipation, participants viewed the 23 experimental trials in three conditions. These trials were created from data from 10 matches involving 14 righthanded players, in various rounds of the tournament. Trials were edited to display one (short sequence length condition), three (medium), or five (long) shots in the sequence preceding the occluded shot, such that they viewed the same final occluded shot three times. The rationale for this approach was that it allowed for reliable comparison of response accuracy scores relative to three sequence lengths (see Figure 2). Altogether, 69 experimental trials were used (three sets of 23). Short, medium and long trials lasted an average of $1.38(S D=$ $.26), 4.18(S D=.38)$, and $6.83(S D=.55)$ seconds respectively.

$<<$ Insert Figure 2 near here $>>$

\section{Materials, Apparatus, and Set-Up}

Test stimuli were projected on to a $4.1 \times 2.3 \mathrm{~m}$ white projector screen (AV Stumpfl, Wallern, Austria) using a NEC PE401H projector (NEC, Tokyo, Japan). Participants held a racket in their hands as if they were about to play a point and stood $5 \mathrm{~m}$ from the screen which allowed for a viewing angle of $3.0^{\circ}-3.9^{\circ}$ subtended by the opposing player. A similar viewing perspective has been used previously (Loffing, Wilkes, \& Hagemann, 2011; Murphy et al., 2016). 


\section{Procedure}

Participants viewed 18 familiarisation and 69 experimental trials. Different rallies were used in the familiarisation trials than the experimental trials. Six sets of randomised experimental trials were created with two participants from each group viewing one of the six sets. Participants viewed three blocks of 23 trials with a one-minute break between blocks. This break was provided to mitigate against boredom or fatigue effects. Each of the 23 trials were presented three times. A yellow circle was displayed on a black screen for 2 seconds prior to the commencement of the trial to indicate where the ball would be once the trial started and a 6 second inter-trial interval was employed. In the experimental trials, the bounce location of the occluded shot landed deep (past the service line) on the left side (left of the centre line) of the court on eight trials, deep on the right side on eight trials, short (before the service line) on the left side on five trials and short on the right side on two trials. When trials were occluded, participants had been instructed to say aloud which quadrant of the court they anticipated the ball would bounce in (e.g., short-left), while additionally swinging their racket as if to return the upcoming shot. The racket was swung to make the task feel more realistic rather than to act as a dependent measure (cf., Roca, Williams, \& Ford, 2014). The order in which participants carried out the verbal and physical response was not controlled. The lead researcher noted the verbal response. Accuracy was measured as the percentage of correct responses relative to actual final ball bounce location of the occluded shot. Participants did not receive feedback after any of the trials.

\section{Data Analysis}

One-sample $t$-tests were conducted to compare accuracy for the skilled and lessskilled participants compared with chance levels. A $2 \times 3$ (Group [skilled, less-skilled] $\times$ Sequence Length [short, medium, long]) MANOVA with repeated measures was conducted, 
with the percentage of correct depth, direction, and combined judgments serving as the dependent variables. The assumptions of MANOVA were met. Depth, direction, and combined accuracy scores were compared across two groups with observations being independent. The 12 cases in each group were greater than the number of dependent variables being analysed. Upon screening for univariate outliers using boxplots and Q-Q plots and multivariate outliers using the Mahalanobis distance, no outliers were detected. The data were further tested for normality. All variables for each group displayed neither significant skewness nor kurtosis and Shapiro-Wilk test of normality was significant only for short combined judgments of skilled participants. Using the recommended alpha level of .001, Box's M test was non-significant, meaning there was homogeneity of variance-covariance matrices. The means of the correlations between depth and combined, direction and combined, and depth and direction accuracy scores were $0.77,0.67$, and 0.25 respectively meaning there was no multicollinearity. The Greenhouse-Geisser correction was applied in the case of violations of sphericity. Partial eta squared $\left(\eta_{p}^{2}\right)$ values are reported for effect size of main effects. The alpha level of statistical significance was set at .05. In the case of multiple $t$-tests, sequential Bonferroni was applied to control for family-wise error. Finally, pairwise comparisons were carried out in the case of significant interactions. To account for the relatively small sample size, a corrected measure of Cohen's $d$ ( $d_{\text {unbiased }}$ as proposed by Cumming, 2012) is reported for effect size of these comparisons. $95 \%$ confidence intervals are reported on $d$.

\section{Results and Discussion}

The mean (and standard error) response accuracy scores for skilled and less-skilled participants for depth, direction, and combined judgment accuracy in short, medium and long sequence length conditions are presented in Figure 3. First, we hypothesised that both skilled 
and less-skilled participants would be more accurate than chance in all conditions, reaffirming previous findings indicating the importance of contextual information in anticipation. As expected, accuracy scores were significantly greater than chance in all conditions (all $p<.01$ ). Participants were able to anticipate accurately based on contextual information alone regardless of skill level.

Second, consistent with our hypothesis that skilled participants would be more accurate than their less-skilled counterparts, a significant main effect of Group was observed, Wilks' Lambda $=.37, F(3,20)=11.53, p<.01, \eta_{p}^{2}=.63$. This main effect was observed for depth, $F(1,22)=25.62, p<.01, \eta_{p}^{2}=.54$, and combined judgments, $F(1,22)=30.55, p<$ $.01, \eta_{p}^{2}=.58$, but not for direction judgments, $F(1,22)=3.36, p=.08, \eta_{p}^{2}=.13$. While a between-groups difference of $4.47 \%$ was observed for direction judgments, the observed differences and associated effect sizes for depth (12.44\%) and combined (15.34\%) judgments were larger. For depth judgments in particular, the motions and/or final positioning of the two players and the ball appears to provide contextual information which upon encoding, allows skilled participants to retrieve relevant alternatives to make more accurate depth judgments than less-skilled counterparts.

Third, the multivariate output revealed no significant effect of Sequence Length on response accuracy. However, because the only other study (Crognier \& Féry, 2005) to our knowledge examining this issue previously in tennis revealed that direction accuracy specifically was increased when a sequence of shots was played prior to the final occluded shot, we examined the univariate output. We report a significant main effect of Sequence Length for direction, $F(2,44)=3.87, p=.03, \eta_{p}^{2}=.15$, but not for depth, $F(2,44)=.53, p=$ $.60, \eta_{p}^{2}=.02$, or combined judgments, $F(2,44)=3.05, p=.06 \eta_{p}^{2}=.12$. Inspection of the 
means and effect sizes associated with pairwise comparisons indicated that the source of the sequence effect for direction judgments was the improvement from short $(M=67.57 \%, S E=$ $1.72)$ to medium $\left(M=73.01 \%, S E=1.74, p=.02, d_{\text {unb }}=0.62,95 \%\right.$ CI $\left.[0.11,1.17]\right)$ trials, with no improvement in accuracy shown from medium to long $\left(p=.87, d_{\mathrm{unb}}=-0.04,95 \% \mathrm{CI}\right.$ $[-0.51,0.43])$ trials. The main effect of Sequence Length for direction judgments and the associated medium effect sizes observed for differences between conditions in which the preceding shot sequence is presented and when it is not, suggest that contextual information can be picked up from the preceding shot sequence to facilitate anticipation. Moreover, the increase in accuracy from short to medium trials with no further increase on long trials suggests that the important information is contained in the shots immediately preceding the final occluded shot rather than earlier shots in the sequence.

The Skill $\times$ Sequence Length interaction was not significant at the multivariate or univariate levels; however, Figure 1 shows that skilled participants were more accurate in their direction judgments on medium $\left(M=75.72 \%, S E=2.70 ; p=.02, d_{\mathrm{unb}}=0.92,95 \% \mathrm{CI}\right.$ $[0.04,1.90])$ and long $\left(M=76.09 \%, S E=2.36 ; p=.01, d_{\mathrm{unb}}=1.05,95 \% \mathrm{CI}[0.25,1.98]\right)$ compared with short trials $(M=68.12 \%, S E=1.64)$. Accuracy did not increase significantly from medium to long trials ( $\left.p=.46, d_{\mathrm{unb}}=0.04,95 \% \mathrm{CI}[-0.79,0.87]\right)$. On the other hand, no significant differences in accuracy were observed between short $(M=67.03 \%, S E=3.10)$ and medium $\left(M=70.29 \%, S E=1.99, p=.13, d_{\mathrm{unb}}=0.34,95 \% \mathrm{CI}[-0.26,0.97]\right)$, short and long $\left(M=69.20 \%, S E=2.80, p=.28, d_{\mathrm{unb}}=0.20,95 \% \mathrm{CI}[-0.47,0.89]\right)$, or medium and long trials $\left(p=.33, d_{\mathrm{unb}}=-0.12,95 \%\right.$ CI $\left.[-0.69,0.44]\right)$ for the less-skilled group. The large effect sizes for the differences between short and medium or long trials for skilled participants and the small effect sizes for differences in the accuracy scores of less-skilled participants, at an exploratory level at least, indicate that skilled participants may be able to use contextual information picked up from the preceding shot sequence more effectively than 
less-skilled participants. We tentatively suggest that skilled participants were able to use the contextual information picked up from the shots immediately preceding the occluded shot to access relevant alternatives from LTM, adjusting their expectancies of likely event outcomes and increasing accuracy relative to when this information was not available.

$<<$ Insert Figure 3 near here $>>$

In summary, in Experiment 1, we demonstrated that skilled participants can use contextual information picked up from the preceding shot sequence to accurately anticipate and that the useful information appears to be contained in shots immediately preceding the critical event rather than in earlier shots. However, performers have been shown to rely on different sources of information to varying extents, dependent on the task constraints (Roca et al., 2013). It is therefore likely that in some instances contextual information other than that gleaned from the preceding shot sequence may be of greater utility. In Experiment 2, we therefore further investigate the sources of contextual information which can be used to facilitate anticipation, particularly focusing on ball and player motion and positioning.

\section{Experiment 2}

In an initial attempt to determine the sources of contextual information facilitating anticipation independent of pertinent postural cues, Murphy et al. (2016) collected verbal reports from skilled and less-skilled participants when viewing the type of animations used in Experiment 1. Participants referred more often to the shot placement throughout the rally than anything else and skilled participants referred more often to court geometry (availability of spaces and angles between players) and shot type (e.g., volley, forehand) than less-skilled participants. Although verbal reports provide researchers with access to the conscious thoughts of participants when performing a task, non-verbal and non-propositional information may be difficult for participants to articulate, resulting in incomplete 
verbalizations (Ericsson \& Simon, 1980). As an alternative, some researchers have collected confidence ratings from participants taking part in anticipation tasks to assess their awareness of the information they use to anticipate (Jackson \& Mogan, 2007; Smeeton \& Williams, 2012).

According to Rosenthal's (2000) higher-order thought hypothesis, if a performer has a higher-order thought about the mental state he/she is in, that state can be assumed to be a conscious mental state. Chan (1992) suggested that, in judgment tasks, high levels of accuracy associated with high confidence (the higher order thought) would indicate subjective awareness of the information being used to make accurate judgments, whereas low levels of confidence associated with high levels of accuracy would indicate a lack of subjective awareness. Confidence ratings, collected in conjunction with accuracy data can therefore provide an indication of the task-relevance of the presented information.

Jackson and Mogan (2007) assessed awareness of the sources of information tennis players were consciously using to inform anticipation of whether an opponent would serve to the left, middle, or right of the service box. The authors recorded the confidence levels of participants in their judgments following each trial. A decrement in performance when the ball was occluded compared to a no occlusion condition indicated that the ball (as it is tossed in the air prior to the serve being hit) is a useful source of information when anticipating serve direction. In studies of deception, researchers have shown that performers are more confident when viewing deceptive than non-deceptive actions of opponents (Jackson, Warren, \& Abernethy, 2006; Smeeton \& Williams, 2012), indicating that performers consciously use misleading information intentionally presented by the opponent. In each of these studies as well as in other research where accuracy scores and confidence ratings have been recorded (e.g.,Salmela \& Fiorito, 1979; Tenenbaum, Levy-Kolker, Sade, Lieberman, \& 
Lidor, 1996) participants became more confident as more information was presented via reduced occlusion conditions.

In this experiment, we determined the extent to which player and ball motion and positioning provide contextual information which can facilitate anticipation and the sources of contextual information that tennis players consciously use when constrained to anticipate based on contextual information alone. We compared the accuracy and confidence levels of skilled and less-skilled tennis players when viewing animated footage of sequences of shots occluded on the sixth shot (the long condition in Experiment 1), which were viewed in a dynamic or still display condition, where the still display condition was presented as the final frame of the sequence at the opponent's racket-ball contact. These two display conditions were further presented in three conditions in which either the players (represented by a blue and a red cylinder with rackets not being visible, as in Experiment 1), the ball, or both the players and the ball, were depicted.

First, based on the findings of Murphy et al. (2016), and other studies (e.g., Loffing \& Hagemann, 2014; Loffing, Sölter, Hagemann, \& Strauss, 2016) proposing the importance of player positioning in providing contextual information, we hypothesised that participants would anticipate at higher than chance levels in conditions in which the players were depicted. We further hypothesised, based on the findings of the first experiment and those of Murphy et al. (2016), that skilled participants would be more accurate than less-skilled participants. Second, based on research in which the spatial occlusion paradigm has been employed (e.g., Abernethy \& Russell, 1987; Jackson \& Mogan, 2007; Müller et al., 2010), we hypothesised that accuracy scores would be highest in the condition depicting both the players and the ball but that differences between this condition and other conditions would provide an indication of the relative importance of ball and player motion and positioning as information sources. Third, based on previous research which suggests that skilled tennis 
players use their domain-specific experience and knowledge to help them pick up and utilise contextual information from player positioning more effectively than less-skilled players (Loffing \& Hagemann, 2014), we hypothesised that between-groups differences in accuracy would be more pronounced in conditions in which the players were visible than when only the ball was presented. Finally, based on the findings of Williams et al. (2012) and Sebanz and Shiffrar (2009), we hypothesised that accuracy scores would be higher in the dynamic than still display condition indicating the importance of motion information in the lead up to the event over and above mere positioning information at the moment of occlusion.

Additionally, we explored the relationship between accuracy and confidence relative to the information presented. We expected, based on the research of Chan (1992), a positive relationship between confidence and accuracy to indicate subjective awareness of the sources of information being used to anticipate accurately. We hypothesised, based on the findings of previous studies on anticipation in tennis (Jackson \& Mogan, 2007; Tenenbaum et al., 1996), that no differences in judgment confidence would be observed between groups. We further hypothesised that confidence levels would be highest in the condition depicting both players and the ball as the most information would be available in that condition (Jackson \& Mogan, 2007; Smeeton \& Williams, 2012; Tenenbaum et al., 1996). Finally, we hypothesised that participants would be more confident in conditions in which more information was available for processing (e.g., when both players and ball are depicted or when viewing dynamic footage) compared to when less information is available (Jackson \& Mogan, 2007; Salmela \& Fiorito, 1979; Tenenbaum et al., 1996). 


\title{
Method
}

\section{Participants}

\author{
Altogether, 12 skilled $\left(M_{\text {age }}=25.8, S D=3.7\right)$ and 12 less-skilled $\left(M_{\text {age }}=22.7, S D=\right.$ \\ 3.9) male tennis players with a mean of $20.6(S D=4.4)$ and $5.9(S D=4.9)$ years of tennis \\ playing experience participated. Skilled participants held British Tennis ratings of between \\ 1.1 and 4.1, whereas less-skilled participants held ratings of 10.2 or did not hold a rating. \\ Skilled participants reported having played a mean of $43.2(S D=18.9)$ matches per year, \\ whereas less-skilled participants did not play competitively. One participant in the less- \\ skilled group and two in the skilled group were left-handed players and the rest were right- \\ handed. Participants had normal or corrected vision and those with corrected vision wore \\ glasses or contact lenses while participating. In total, eight of the skilled and seven of the \\ less-skilled participants had taken part in Experiment 1 with the time between the two \\ experiments being approximately four to six months. The research was carried out in line \\ with the lead university's research ethics guidelines, with participants providing informed \\ consent prior to taking part and being aware that they could withdraw from testing at any \\ time without consequence.
}

\section{Test Stimuli}

The longest condition in Experiment 1 (five shots prior to the occluded shot) yielded the highest accuracy scores and consequently, we used this length sequence in the current experiment. Altogether, 21 of the 23 sequences used in Experiment 1 were presented as experimental test stimuli. One trial was omitted because it yielded combined accuracy scores of $0 \%$ in long trials in Experiment 1 . Another trial was excluded because the near player was not visible at the moment of occlusion as he was too far to the side of the court. The outcome of the first of these trials was short on the left side of the court, and was short on the right side 
for the latter. This yielded a remaining eight trials for which the outcome was deep on the left and deep on the right side of the court, four trials for which the outcome was short on the left and one trial for which the outcome was short on the right side.

Participants viewed each of the 21 trials either as moving videos (Dynamic display condition) or as a still image of the final frame of the video (Still display condition). Three presentation conditions were employed: Full (both players and the ball were presented); Players (only the players were presented); and Ball (only the ball was presented). The Players and Ball conditions were created in the same way as described in Experiment 1, with either player movement or ball trajectory data omitted to create the required stimuli. In total, participants viewed each trial in six conditions (Dynamic Full, Dynamic Players, Dynamic Ball, Still Full, Still Players, and Still Ball). All dynamic trials displayed five shots prior to occlusion at the opponent's racket-ball contact on the sixth shot. Still trials were presented for the same amount of time as their corresponding dynamic trials $(M=6.82 \mathrm{~s}, S D=.55)$.

\section{Materials, Apparatus, and Set-Up}

The experimental set-up was the same as in Experiment 1.

\section{Procedure}

Participants viewed a total of 126 experimental (21 trials in each of the 6 conditions) and 18 familiarisation trials. Different rallies were used in the familiarisation trials than the experimental trials. Six sets of randomised trials were created with two participants from each group viewing one of the six sets. Participants viewed four blocks (two of 32, two of 31) of trials, viewing each of the 21 trials six times. A one-minute break was provided between blocks to mitigate against boredom or fatigue effects. Participants responded in the same way as in Experiment 1. In still trials, participants were required to wait for occlusion before 
responding. When viewing the familiarization trials, participants were reminded that it was important not to respond until the point of occlusion. After verbally predicting the outcome of the opponent's shot, participants then rated how confident they were in their combined judgment on a scale of 1 to 5 , with 1 being not at all confident, and 5 being extremely confident. Participants did not receive feedback after any of the trials. An inter-trial interval of six seconds was employed.

\section{Data Analysis}

Response Accuracy

One-sample $t$-tests were conducted to compare depth, direction, and combined judgments to chance levels in each presentation and display condition. Prior to conducting further analyses, we determined whether the assumptions of MANOVA were met. As in Experiment 1, depth, direction, and combined accuracy were continuously compared across two groups, with observations being independent. No significant univariate or multivariate outliers were detected. Significant violations $(p<.05)$ of skewness and kurtosis were observed on five of the 36 variable combinations. Six of the 36 variable combinations violated the Shapiro-Wilk test of normality. No data transformations were deemed necessary given the equal sample size. Since the number of dependent variables was greater than the number of participants in each group, we refrained from conducting a MANOVA. We ran a 2 $($ Group [skilled, less-skilled] $) \times 2($ Display [Dynamic, Still] $) \times 3($ Presentation [Full, Players, Ball]) ANOVA for each of depth, direction, and combined judgments.

\section{Solution Probabilities}

\section{Prior to determining whether there was a correlation between combined response} accuracy and confidence on the 21 individual trials for skilled and less-skilled participants, 
we verified whether the data were normally distributed. No significant outliers were detected. Significant skewness and kurtosis $(p<.05)$ was observed on one of the 24 variable combinations with the Shapiro-Wilk test of normality violated on three of the 24 variable combinations. Therefore, we calculated Spearman's correlation coefficient in each of the six conditions for skilled and less-skilled participants.

\section{Confidence Ratings}

The data were screened to verify the assumptions of ANOVA were met. Confidence ratings were continuously compared across groups and observations were independent. No significant outliers were detected. Neither significant skewness nor kurtosis was observed and the Shapiro-Wilk test of normality was only significant in the Still Players condition for skilled participants. In light of equal sample sizes, transformations were not deemed necessary. A 2 (Group) $\times 2$ (Display) $\times 3$ (Presentation) ANOVA was conducted for confidence ratings of combined judgments.

Partial eta squared $\left(\eta_{p}^{2}\right)$ values are reported throughout for effect size of main effects. In the case of violations of sphericity, the Greenhouse-Geisser correction was applied. The alpha level of statistical significance was set at .05. Sequential Bonferroni adjustments were applied in the case of multiple pairwise comparisons to adjust for family-wise error. Finally, pairwise comparisons were conducted in the case of significant interactions and main effects. $d_{\text {unbiased }}$ is reported for effect size of these comparisons, and 95\% confidence intervals are reported on $d$. 


\section{Results and Discussion}

\section{Response Accuracy}

The mean depth, direction, and combined response accuracy (and standard error) for participants across groups are presented in Figure 4. We hypothesised, based on previous research suggesting the potential importance of player positioning as a source of contextual information (e.g., Loffing \& Hagemann, 2014; Murphy et al., 2016), that participants would be more accurate than chance in the two presentation conditions depicting the players. Onesample $t$-tests revealed that skilled participants were significantly more accurate than chance for depth, direction, and combined judgments in all presentation conditions in both dynamic and still format (all $p<.01$ ), except direction judgments in the Still Ball condition, while lessskilled participants were more accurate than chance in all presentation conditions in dynamic and still format (all $p<.05$ ), except for direction and combined judgments in the Still Ball condition. While Murphy et al. (2016) demonstrated that contextual information can be used to anticipate effectively, independently of access to pertinent postural cues, these findings indicate that enough contextual information can be picked up solely from the motions and final positions of the players or the ball (although the final position of the ball does not appear sufficient for making direction judgments) to facilitate anticipation.

As in Experiment 1, and in support of Murphy et al. (2016), a significant main effect of Group was observed for depth, $F(1,22)=15.98, p<.01, \eta_{p}^{2}=.42$ and combined judgments, $F(1,22)=24.55, p<.01, \eta_{p}^{2}=.53$ but not direction judgments, $F(1,22)=3.73, p$ $=.07, \eta_{p}^{2}=.15$. Larger between groups differences of $11.38 \%$ and $12.77 \%$ were observed for depth and combined judgments, in comparison to direction judgments (4.56\%). 
Second, we hypothesised, based on previous research employing the spatial occlusion paradigm (e.g., Abernethy \& Russell, 1987; Jackson \& Mogan, 2007), that accuracy would be highest in the Full Presentation condition. A significant main effect of Presentation was observed for depth, $F(2,44)=4.29, p=.02, \eta_{p}^{2}=.16$, direction, $F(2,44)=70.88, p<.01, \eta_{p}^{2}$ $=.76$, and combined judgments, $F(2,44)=33.71, p<.01, \eta_{p}^{2}=.61$. The effect sizes imply a larger effect of Presentation condition for direction and combined than depth judgments. Combined accuracy scores were lower in the Ball $(M=35.71 \%, S E=1.14)$, compared to the Players $\left(M=48.31 \%, S E=2.30, p<.01, d_{\mathrm{unb}}=0.91,95 \%\right.$ CI $\left.[0.50,1.34]\right)$ and Full conditions $\left(M=51.39 \%, S E=1.58, p<.01, d_{\mathrm{unb}}=1.18,95 \%\right.$ CI $\left.[0.80,1.60]\right)$, with accuracy in the Players and Full conditions not differing significantly $\left(p=.11, d_{\mathrm{unb}}=0.20,95 \% \mathrm{CI}[-\right.$ $0.05,0.45])$. The decrement in performance when the motion and final positioning of the players was omitted from the display is indicative of their importance as an information source. A similar pattern was observed for direction (Ball: $M=54.56 \%, S E=1.26$; Players: $M=72.82, S E=1.82 ;$ Full: $M=71.73, S E=1.50)$ with significant differences observed between the Full and the Ball conditions $\left(p<.01, d_{\mathrm{unb}}=1.27,95 \% \mathrm{CI}[0.87,1.72]\right)$ and between the Players and Ball conditions $\left(p<.01, d_{\mathrm{unb}}=1.40,95 \%\right.$ CI $\left.[0.94,1.89]\right)$ but not the Full and Players conditions ( $p=.42, d_{\text {unb }}=-0.09,95 \%$ CI $\left.[-0.36,0.18]\right)$. However, mean differences were much smaller for depth (Ball: $M=68.25 \%, S E=1.62$; Players: $M=66.17$, $S E=1.92$; Full: $M=70.54, S E=1.40)$ for which comparatively high scores were observed when only the ball was visible. Accuracy scores were significantly higher in the Full than the Players condition $\left(p=.01, d_{\mathrm{unb}}=0.37,95 \% \mathrm{CI}[0.10,0.66]\right)$. No significant differences were observed between the Full and the Ball $\left(p=.10, d_{\text {unb }}=0.20,95 \%\right.$ CI $\left.[-0.05,0.46]\right)$ or the Ball and Players conditions $\left(p=.23, d_{\mathrm{unb}}=0.18,95 \%\right.$ CI $\left.[-0.12,0.49]\right)$. In line with our hypothesis, overall accuracy was highest when both the players and the ball were depicted suggesting these sources are most useful when presented in tandem. Nevertheless, more 
pronounced decrements in performance when the players were omitted from the display indicate that, particularly for direction judgments, the motion and/or final positioning of the players is a more important source of contextual information than that of the ball.

A significant Display $\times$ Presentation interaction was observed for direction, $F(2,44)=$ $15.86, p<.01, \eta_{p}^{2}=.42$, and combined judgments, $F(2,44)=7.78, p<.01, \eta_{p}^{2}=.26$, but not depth judgments, $F(2,44)=.55, p=.58, \eta_{p}^{2}=.02$. Follow-up pairwise comparisons revealed that the direction and combined differences between Still and Dynamic display conditions were significant for the Ball condition (direction: $p<.01, d_{\mathrm{unb}}=1.67,95 \% \mathrm{CI}[0.90,2.53]$, combined: $p<.01, d_{\mathrm{unb}}=1.16,95 \%$ CI $\left.[0.51,1.87]\right)$, but not for the Players or Full conditions. We further investigated the interaction separately for the Dynamic and Still display conditions. In the Dynamic condition a main effect of Presentation was significant for depth, $F(2,44)=3.60, p=.04, \eta_{p}^{2}=.14$, direction, $F(2,44)=8.33, p<.01, \eta_{p}^{2}=.28$, and combined judgments, $F(2,44)=7.81, p<.01, \eta_{p}^{2}=.26$ but no significant interactions were observed. The direction accuracy scores were higher in the Dynamic Full $(M=71.83, S E=$ $\left.1.87, p<.01, d_{\mathrm{unb}}=.77,95 \% \mathrm{CI}[0.30,1.27]\right)$ and Dynamic Players $(M=71.43, S E=2.01, p$ $\left.=.01, d_{\mathrm{unb}}=.73,95 \% \mathrm{CI}[0.22,1.26]\right)$ conditions than in the Dynamic Ball $(M=63.49, S E=$ 2.39) condition. The combined accuracy scores were higher in the Dynamic Full $(M=51.19$, $S E=2.47)$ than the Dynamic Ball condition only $\left(M=41.47, S E=2.10, p=.01, d_{\mathrm{unb}}=.84\right.$, $95 \%$ CI $[0.39,1.33])$. No significant differences were observed for depth judgments. In the Still condition a main effect of Presentation was significant for direction, $F(2,44)=59.08, p$ $<.01, \eta_{p}^{2}=.73$ and combined judgments, $F(2,44)=27.52, p<.01, \eta_{p}^{2}=.56$ but not depth $F(2,44)=49.13, p=.46, \eta_{p}^{2}=.04$ and no significant interactions were observed. The accuracy scores for direction were higher in the Still Full $\left(M=71.63, S E=3.24, p<.01, d_{\text {unb }}\right.$ 
$=1.96,95 \%$ CI $[1.27,2.76])$ and Still Players $\left(M=74.21, S E=2.64, p<.01, d_{\mathrm{unb}}=2.34\right)$ conditions than in the Still Ball $(M=45.64, S E=1.87)$ condition. Also, the combined accuracy scores were higher in the Still Full $\left(M=51.59, S E=3.22, p<.01, d_{\mathrm{unb}}=1.53,95 \%\right.$ CI $[0.93,2.22])$ and Still Players $\left(M=50.40, S E=3.37, p<.01, d_{\mathrm{unb}}=1.38,95 \%\right.$ CI $[0.74$, 2.09]) conditions than in the Still Ball $(M=29.96, S E=1.80)$ condition. These findings suggest that, when making direction judgments in particular, contextual information picked up from the motion and final positioning of the players facilitates anticipation more so than the motion and final positioning of the ball.

We hypothesised that between groups differences would be stronger when the motion and positioning of the players was depicted than when only the ball was visible. However, the Group $\times$ Presentation interaction was not significant for depth, $F(2,44)=1.65, p=.20, \eta_{p}^{2}=$ .07 , direction, $F(2,44)=1.14, p=.33, \eta_{p}^{2}=.05$, or combined, $F(2,44)=3.04, p=.06, \eta_{p}^{2}=$ .12 , judgments. The increase in combined accuracy from the Ball (Skilled: $M=39.29 \%, S E=$ 1.61; Less-skilled: $M=32.14, S E=1.61)$ to the Players (Skilled: $M=55.56 \%, S E=3.25$; Less-skilled: $M=41.07, S E=3.25$ ) and Full (Skilled: $M=59.72 \%, S E=2.23$; Less-skilled: $M=43.06, S E=2.23$ ) conditions was greater for skilled than less-skilled participants. These data, however, do not conclusively imply that skilled participants use contextual information picked up from the motion and positioning of the players to anticipate more accurately than less-skilled participants.

Third, based on the findings of Williams et al. (2012) and Sebanz and Shiffrar (2009), we hypothesised that accuracy scores would be higher in the Dynamic than the Still display condition. In contrast to our hypothesis, the main effect of Display was not significant for depth, $F(1,22)=.28, p=.60, \eta_{p}^{2}=.01$, direction, $F(1,22)=3.23, p=.09, \eta_{p}^{2}=.13$, or 
combined judgments, $F(1,22)=.85, p=.37, \eta_{p}^{2}=.04$. This finding was surprising given that a main effect of Sequence Length was observed for direction judgments in Experiment 1. One would therefore expect that in this experiment participants would be more accurate when the preceding shot sequence was presented (in the Dynamic Display condition) than when it was not (in the Still Display condition). The final positioning of the players and the ball at racket-ball contact (particularly when presented together) potentially yield as much information as when the motion of the players and the ball in the lead up to racket-ball contact is also available. No other significant main effects or interactions were observed.

$<<$ Insert Figure 4 near here $>>$

\section{Solution Probabilities}

The scatterplots of the correlations between combined response accuracy and confidence ratings are presented in Figure 5. To determine the relationship between accuracy and confidence levels on the 21 individual trials, and thus determine the extent to which participants were aware of the information they were using to inform their judgments (Chan, 1992), we calculated Spearman's correlation coefficients in each of the 6 conditions for skilled and less-skilled participants. There was a strong significant correlation $\left(r_{s}=.64, p<\right.$ .01) between combined accuracy and confidence in the Dynamic Full condition for skilled participants only. Because the skilled participants were highly confident in their more accurate judgments and less confident in their less accurate judgments in this condition only, we suggest that they consciously use a combination of player and ball motion when making these judgments (Chan, 1992). Skilled participants were also more accurate than less-skilled participants when making judgments in this condition $\left(p<.01, d_{\mathrm{unb}}=2.24,95 \% \mathrm{CI}[1.25\right.$, 3.35]). It therefore appears that both the motion of the players and the ball in the lead up to 
the opponent's racket-ball contact are required for skilled performers to be aware of the information they should attend to in order to anticipate highly accurately.

$<<$ Insert Figure 5 near here $>>$

\section{Confidence Ratings}

The mean confidence levels (and standard error) of skilled and less-skilled participants for each display and presentation condition are presented in Figure 6. First, based on the findings of Jackson and Mogan (2007), we hypothesised that confidence would not differ across the two groups. A 2 (Group) $\times 2$ (Display) $\times 3$ (Condition) ANOVA revealed that the main effect of Group was non-significant, $F(1,22)=3.77, p=.07, \eta_{p}^{2}=.15$. The large effect size reflected skilled participants being more confident $(M=2.84, S E=.15)$ overall than their less-skilled counterparts $(M=2.44, S E=.15)$. Although not statistically significant, the direction of the effect is in contrast with the findings of both Tenenbaum et al. (1996) and Jackson and Mogan (2007) who reported lower levels or levels of confidence that did not differ respectively in skilled compared to less-skilled tennis players when judging the direction of tennis serves. However, the higher confidence ratings of the skilled group in this experiment reflect the overall higher accuracy scores they made in comparison to the lessskilled participants.

Second, we hypothesised that participants would be more confident in the dynamic than the still display condition. A significant main effect of Display, $F(1,22)=18.12, p<$ $.01, \eta_{p}^{2}=.45$, was observed. The confidence ratings were higher when viewing the Dynamic display $(M=2.83, S E=.11)$ than the Still display $(M=2.45, S E=.11)$. Although the expected significant effect of Display was not observed for accuracy, participants appear to 
consider player and ball information presented in a dynamic manner to be more useful to informing their judgments than just the final positioning of these elements.

Third, we hypothesised that participants would be most confident when viewing the Full display conditions. A significant main effect of Presentation condition was observed, $F(2,44)=43.46, p<.01, \eta_{p}^{2}=.66$. Participants were more confident $(p<.01)$ in the Full condition $(M=3.01, S E=.12)$ than in the Players $(M=2.48, S E=.12)$ and Ball conditions $(M=2.43, S E=.10)$. This supports the findings of Jackson and Mogan (2007), who found that participants were more confident when anticipating serves in conditions of no occlusion in comparison to when certain parts of the opponent's body or equipment were occluded (cf., Tenenbaum et al., 1996).

Finally, a significant Display $\times$ Presentation interaction was observed, $F(1.47,32.4)=$ 25.23, $p<.01, \eta_{p}^{2}=.53$. Follow-up pairwise comparisons revealed that participants were more confident when viewing the Dynamic Ball $(M=2.82, S E=.12)$ than the Still Ball condition $\left(M=2.05, S E=.13, p<.01, d_{\mathrm{unb}}=1.19,95 \% \mathrm{CI}[0.69,1.75]\right)$ and when viewing the Dynamic Full $(M=3.13, S E=.12)$ than the Still Full condition $(M=2.89, S E=.13, p=$ $.01, d_{\mathrm{unb}}=0.36,95 \%$ CI $\left.[0.06,0.69]\right)$, although the effect size was much smaller for this difference. The difference in confidence between the Dynamic Players $(M=2.54, S E=.13)$ and Still Players $(M=2.42, S E=.13)$ conditions was non-significant. In conjunction with the accuracy findings in the Still Ball condition, the large effect size observed between Dynamic and Still Ball conditions further indicates that participants do not consider final ball position to be a useful source of information on its own for making accurate anticipation judgments. Conversely, participants appear to find the final positioning of the two players to be just as useful as when the motion of the two players is presented throughout the rally. No other significant main effects or interactions were observed. 
THE ROLE OF CONTEXT IN ANTICIPATION

$<<$ Insert Figure 6 near here $>>$

\section{General Discussion}

We examined the extent to which specific sources of contextual information (shot sequencing, ball and player motion and positioning) facilitate anticipation. In Experiment 1, we examined whether the shot sequence preceding the to-be-anticipated shot could be used to facilitate anticipation by presenting skilled and less-skilled participants with sequences of shots of varying length. In Experiment 2, we investigated whether ball and player motion and positioning could be used to facilitate accurate anticipation by presenting footage which depicted the players, the ball or both in dynamic or still form, as well as investigating participant awareness of the information used to respond, reflected in confidence level.

In line with previous findings demonstrating that performers can use contextual information picked up from the preceding sequence of events (e.g., Loffing et al., 2015; Milazzo et al., 2015), participants became more accurate in their direction judgments when presented with the shot sequence preceding the occluded shot. This finding extends previous research by demonstrating that the preceding shot sequence provides contextual information which can be used to accurately anticipate the direction of an opponent's shot independent of postural information. Moreover, we observed that the important contextual information picked up from the preceding shot sequence appears to be contained in the shots immediately preceding the critical event. Intuitively, at least, this seems to make sense, in that in dynamic sports like tennis, the situation can change drastically from one shot to the next. These findings have implications for the design of testing and training protocols as well as for the level of detail that should be reported by researchers about the test stimuli used to conduct studies. Previously, researchers have considered it sufficient to merely report the duration of trials prior to occlusion (e.g., North et al., 2011; Gorman et al., 2013; Ward, Ericsson, \& 
Williams, 2013). We suggest that in future researchers, should also report the number of actions or events in the preceding sequence such that the extent to which this information may contribute to the judgments being made can be inferred.

While we expected that skilled participants would be able to use contextual information picked up from the preceding shot sequence to anticipate more effectively than less-skilled counterparts, the Group $\times$ Sequence Length interaction was not significant. We suggest that the sequential patterns emerging from the preceding shots were not complex enough to clearly differentiate between skill groups. Loffing et al. (2015) found that task complexity affected whether the judgments of less-skilled participants were influenced by sequential patterns in a volleyball anticipation task. Initially, only skilled volleyball players were influenced by sequential patterns, whereas when the task difficulty was reduced, the less-skilled players were also influenced. As a result of the vast amount of experience and practice accumulated, skilled performers have built up large knowledge bases, which can be drawn upon to adjust their expectancies of potential event outcomes in domain-specific situations. It is likely that in addition to general patterns of play which potentially apply in many sports, more complex domain-specific patterns of play exist (McGarry \& Franks, 1996) which require domain-specific knowledge to be detected and subsequently used to facilitate anticipation. It is possible that the test stimuli used in this study included more simple rather than complex patterns, decreasing the likelihood of skill differences being observed.

The contextual information gleaned from the players was observed to be more useful than the ball for making accurate anticipation judgments. Combined accuracy was lowest in the Ball condition with no differences being observed in combined accuracy between the Full and Players conditions. The increase in accuracy as a result of including the ball in the display in addition to the players was therefore minimal. Loffing et al. (2011) reported similar findings when ball flight information was presented in addition to the opponent's movement 
pattern and court positioning. Although the already high direction accuracy scores increased when ball flight information was made available, this increase was not statistically significant. It appears that while the ball contributes to accurate anticipation, it does not provide as much pertinent contextual information as the motion and/or positioning of the players, which appear to provide highly important information without which severe decrements in anticipation occur.

Although the ball was shown not to be as useful an information source as the players, participants were nevertheless able to pick up some useful information from its motion. Specifically, accuracy significantly increased from the Still to the Dynamic display condition when only the ball was presented. In contrast, no differences in display condition were found when only the players or both the players and the ball were presented. While it appears that as much useful contextual information may be picked up from the final position of the players as when the preceding motion of the players are also available, the useful contextual information picked up from the ball comes from its flight. Moreover, given that direction rather than depth accuracy increased when the preceding shot sequence was presented in Experiment 1 and it is direction accuracy, again, which increases when the motion of the ball is presented rather than just its final position, we tentatively suggest that it is this information that is picked up from the ball flight when its motion is presented.

The somewhat lower accuracy in the Dynamic Ball in comparison to the Dynamic Full condition nevertheless suggests that participants integrate all available sources of relevant information to make accurate judgments. The confidence data support this notion, in that confidence levels were highest when both the players and the ball were presented. Moreover, the positive correlation between confidence ratings and accuracy for the skilled participants in the Dynamic Full condition suggests that only when both the players and the ball are depicted are they aware of the information they are using to anticipate effectively. 
Ward et al. (2013) and Belling, Suss, and Ward (2015) suggest, based on Long Term Working Memory theory (Ericsson \& Kintsch, 1995), that when presented with a domainspecific situation, skilled performers have access to more task-relevant and fewer taskirrelevant options than less-skilled performers, which ultimately results in more accurate judgments. The Dynamic Full condition most closely mimics the conditions in which the skilled participants in this study would have built up large amounts of experience and practice. It is therefore possible that only when both the motion of the players and the ball are presented can skilled participants consciously attend to, and encode, this information in a manner in which it can be associated with an appropriate retrieval cue to facilitate access to task-relevant options from LTM.

Because direction accuracy was higher when the preceding shot sequence was presented in Experiment 1 than when it was not, we expected a similar effect when participants viewed dynamic animations containing the preceding shot sequence compared to when they viewed only a still image in Experiment 2. In contrast to our hypothesis, and the findings of Williams et al. (2012), this effect was not significant. Gorman et al. (2013) observed that performance levels were higher in a static than a dynamic basketball pattern recall and decision making task. The authors suggested that although dynamic sequences contain more information (via the build-up of actions in the preceding sequence), a combination of the shorter viewing time of the final frame of the dynamic sequences $(.03$ seconds in our study) in comparison to when viewing the still images (approximately 7 seconds in our experiment), and the complex nature of the dynamic sequences may have had a detrimental effect on performance. The duration of the still trials in Experiment 2 is therefore a potential limitation in our approach. However, although higher confidence levels when viewing dynamic sequences rather than still images indicate that dynamic sequences may be perceived to contain more useful information than a still image of the critical event, if 
these still images are presented for a long enough period of time, useful contextual information can be picked up from the positioning of the players to anticipate as accurately as if more information is presented in a dynamic manner. The practical significance of this effect is questionable because in real-world dynamic tasks this information is usually only presented for a very a short period of time. Perhaps the message is that in dynamic tasks the final positions of the various elements in the environment contain useful information which, if the rapid extraction of this information can be trained, could facilitate anticipation.

Another potential limitation of the study is that the laboratory setting and response modality we employed do not directly correspond with what would be found in a real-world setting. The verbal response used, decouples perception and action and therefore the extent to which these findings would transfer to a real-world setting is not clear (Dicks, Button, \& Davids, 2010; van der Kamp, Rivas, van Doorn, \& Savelsbergh, 2008). Of particular relevance to the interpretation of our findings, in the context of anticipatory expertise, Mann, Farrow and Abernethy (2010) demonstrated that when anticipating the direction of a cricket bowl visually occluded at hand-ball release, skilled batters but not novices increased response accuracy as a function of the degree of perception-action coupling involved. Moreover, of the four response modalities (verbal response, foot movement, shadow batting and batting) employed, a significant expert anticipatory advantage was only observed in the shadow batting and batting conditions (i.e., those conditions with the highest levels of action specificity). These findings suggests that the nature of the expert anticipatory advantage may not be fully revealed in experimental tasks which do not closely mimic the task demands experienced in real-world settings. We therefore recommend that, in future, researchers strive to create representative tasks which can more fully examine the expert anticipatory advantage as well as verify the extent to which the findings of less representative, yet highly controlled, tasks generalize to the natural environment. 
Additionally, when playing tennis, players would have a first person perspective and therefore only see one player (the opponent) rather than two players (as in our stimuli). While numerous other researchers have successfully employed a similar viewing perspective or methodological approach (e.g., Gorman et al., 2013; Vaeyens, Lenoir, Williams, Mazyn, \& Philippaerts, 2007; Williams \& Davids, 1998; Williams et al., 2012) in studies on anticipation, decision making and pattern perception, we recommend that in future, researchers continue to create experimental set-ups which more closely represent the realworld environment (e.g., Runswick, Roca, Williams, Bezodis, \& North, 2017). Nevertheless, we do believe that in some instances, doing so may not be possible, or indeed may not present the best approach to adopt. For example, given that our aim in this paper was to determine the extent to which specific sources of contextual information facilitate anticipation independent of pertinent postural cues, it is likely that technological limitations would have made eliminating such cues in a field-based study impossible. Moreover, in our current approach, depicting both players was necessary to determine the importance of player motion and positioning in particular. Additionally, differences across skill groups were observed in this paper, providing a modicum of construct validity for the approach.

In sum, we examined the extent to which specific sources of contextual information could be used by skilled and less-skilled tennis players to make accurate judgments when constrained to anticipate the outcome of an opponent's shot independent of pertinent postural cues. We report novel findings indicating that when presented with the shot sequence preceding the to-be-anticipated shot, direction but not depth accuracy was positively affected. We further demonstrated that the motion and positioning of the players appear to provide more useful contextual information than the ball flight and that only when both player and ball motion is available in the lead up to the critical event are skilled performers aware of the information they use to inform their judgments. It appears that rather than acting 
independently, all of the sources of contextual information which would normally be available to performers interact, and through experience and practice can be utilised to effectively facilitate highly accurate anticipation judgments in skilled performers. 


\section{References}

Abernethy, B. (1990). Expertise, visual search, and information pick-up in squash. Perception, 19(1), 63-77.

Abernethy, B., Gill, D., Parks, S., \& Packer, S. (2001). Expertise and the perception of kinematic and situational probability information. Perception, 30(2), 233-252.

Abernethy, B., \& Russell, D. G. (1987). The relationship between expertise and visual search strategy in a racquet sport. Human Movement Science, 6(4), 283-319.

Abernethy, B., \& Zawi, K. (2007). Pickup of essential kinematics underpins expert perception of movement patterns. Journal of Motor Behavior, 39(5), 353-367.

Abernethy, B., Zawi, K., \& Jackson, R. C. (2008). Expertise and attunement to kinematic constraints. Perception, 37(6), 931-948.

Belling, P., Suss, J., \& Ward, P. (2015). Advancing theory and application of cognitive research in sport: Using representative tasks to explain and predict skilled anticipation, decision-making, and option-generation behavior. Psychology of Sport and Exercise, 16(1), 45-59.

Buckolz, E., Prapavesis, H., \& Fairs, J. (1988). Advance cues and their use in predicting tennis passing shots. Canadian Journal of Sport Sciences $=$ Journal Canadien Des Sciences Du Sport, 13(1), 20.

Cañal-Bruland, R., \& Williams, A. M. (2010). Recognizing and predicting movement effects: Identifying critical movement features. Experimental Psychology, 57(4), 320-326. 
Causer, J., Smeeton, N. J., \& Williams, A. M. (2017). Expertise differences in anticipatory judgements during a temporally and spatially occluded task. PloS One, 12(2), e0171330.

Chan, C. (1992). Implicit Cognitive Processes: Theoretical Issues and Applications in Computer Systems Design. Unpublished doctoral dissertation, University of Oxford, England.

Crognier, L., \& Féry, Y. (2005). Effect of tactical initiative on predicting passing shots in tennis. Applied Cognitive Psychology, 19(5), 637-649.

Cumming, G. (2012). Understanding the new statistics: Effect sizes, confidence intervals, and meta-analysis. Routledge.

Dicks, M., Button, C., \& Davids, K. (2010). Examination of gaze behaviors under in situ and video simulation task constraints reveals differences in information pickup for perception and action. Attention, Perception, \& Psychophysics, 72(3), 706-720.

Ericsson, K. A., \& Kintsch, W. (1995). Long-term working memory. Psychological Review, 102(2), 211-245.

Ericsson, K. A., \& Simon, H. A. (1980). Verbal reports as data. Psychological Review, 87(3), 215.

Ericsson, K. A., Patel, V., \& Kintsch, W. (2000). How experts' adaptations to representative task demands account for the expertise effect in memory recall: Comment on Vicente and Wang (1998). Psychological Review, 107, 578-592.

Farrow, D., \& Reid, M. (2012). The contribution of situational probability information to anticipatory skill. Journal of Science and Medicine in Sport, 15(4), 368-373. 
Gorman, A. D., Abernethy, B., \& Farrow, D. (2011). Investigating the anticipatory nature of pattern perception in sport. Memory \& Cognition, 39(5), 894-901.

Gorman, A. D., Abernethy, B., \& Farrow, D. (2012). Classical pattern recall tests and the prospective nature of expert performance. The Quarterly Journal of Experimental Psychology, 65(6), 1151-1160.

Gorman, A. D., Abernethy, B., \& Farrow, D. (2013). Is the relationship between pattern recall and decision-making influenced by anticipatory recall? The Quarterly Journal of Experimental Psychology, 66(11), 2219-2236.

Gray, R. (2002a). Behavior of college baseball players in a virtual batting task. Journal of Experimental Psychology: Human Perception and Performance, 28(5), 1131-1148.

Gray, R. (2002b). "Markov at the bat": A model of cognitive processing in baseball batters. Psychological Science, 13(6), 542-547.

Huys, R., Cañal-Bruland, R., Hagemann, N., Beek, P. J., Smeeton, N. J., \& Williams, A. M. (2009). Global information pickup underpins anticipation of tennis shot direction. Journal of Motor Behavior, 41(2), 158-170.

Jackson, R. C., \& Mogan, P. (2007). Advance visual information, awareness, and anticipation skill. Journal of Motor Behavior, 39(5), 341-351.

Jackson, R. C., Warren, S., \& Abernethy, B. (2006). Anticipation skill and susceptibility to deceptive movement. Acta Psychologica, 123(3), 355-371. 
Loffing, F., Sölter, F., Hagemann, N., \& Strauss, B. (2016). On-court position and handedness in visual anticipation of stroke direction in tennis. Psychology of Sport and Exercise, 27, 195-204.

Loffing, F., Stern, R., \& Hagemann, N. (2015). Pattern-induced expectation bias in visual anticipation of action outcomes. Acta Psychologica, 161, 45-53.

Loffing, F., Wilkes, T., \& Hagemann, N. (2011). Skill level and graphical detail shape perceptual judgments in tennis. Perception, 40(12), 1447.

Loffing, F., \& Hagemann, N. (2014). On-court position influences skilled tennis players' anticipation of shot outcome. Journal of Sport \& Exercise Psychology, 36(1), 14-26.

Mann, D. L., Abernethy, B., \& Farrow, D. (2010). Action specificity increases anticipatory performance and the expert advantage in natural interceptive tasks. Acta Psychologica, 135(1), 17-23.

McGarry, T., \& Franks, I. M. (1996). In search of invariant athletic behaviour in sport: An example from championship squash match-play. Journal of Sports Sciences, 14(5), 445 456.

McRobert, A. P., Ward, P., Eccles, D. W., \& Williams, A. M. (2011). The effect of manipulating context-specific information on perceptual-cognitive processes during a simulated anticipation task. British Journal of Psychology (London, England : 1953), $102(3), 519-534$.

Milazzo, N., Farrow, D., Ruffault, A., \& Fournier, J. F. (2015). Do karate fighters use situational probability information to improve decision-making performance during onmat tasks? Journal of Sports Sciences, 34(16), 1547-1556. 
Müller, S., Abernethy, B., Eid, M., McBean, R., \& Rose, M. (2010). Expertise and the spatiotemporal characteristics of anticipatory information pick-up from complex movement patterns. Perception, 39(6), 745-760.

Müller, S., \& Abernethy, B. (2012). Expert anticipatory skill in striking sports: A review and a model. Research Quarterly for Exercise and Sport, 83(2), 175-187.

Murphy, C. P., Jackson, R. C., Cooke, K., Roca, A., Benguigui, N., Williams, A. M., (2016). Contextual information and perceptual-cognitive expertise in a dynamic, temporallyconstrained task. Journal of Experimental Psychology: Applied, 22(4), 455-470.

Navia, J. A., van der Kamp, J., \& Ruiz, L. M. (2013). On the use of situational and body information in goalkeeper actions during a soccer penalty kick. International Journal of Sport Psychology, 44(3), 234-251.

North, J. S., Ward, P., Ericsson, A., \& Williams, A. M. (2011). Mechanisms underlying skilled anticipation and recognition in a dynamic and temporally constrained domain. Memory (Hove, England), 19(2), 155-168.

North, J., Williams, A., Hodges, N., Ward, P., \& Ericsson, K. (2009). Perceiving patterns in dynamic action sequences: Investigating the processes underpinning stimulus recognition and anticipation skill. Applied Cognitive Psychology, 23(6), 878-894.

Roca, A., Ford, P. R., McRobert, A. P., \& Williams, A. M. (2011). Identifying the processes underpinning anticipation and decision-making in a dynamic time-constrained task. Cognitive Processing, 12(3), 301-310. 
Roca, A., Ford, P. R., McRobert, A. P., \& Williams, A. M. (2013). Perceptual-cognitive skills and their interaction as a function of task constraints in soccer. Journal of Sport \& Exercise Psychology, 35(2), 144-155.

Roca, A., Williams, A. M., \& Ford, P. R. (2014). Capturing and testing perceptual-cognitive expertise: A comparison of stationary and movement response methods. Behavior Research Methods, 46(1), 173-177.

Rosenthal, D. M. (2000). Consciousness, content, and metacognitive judgments. Consciousness and Cognition, 9(2), 203-214.

Runswick, O. R., Roca, A., Williams, A. M., Bezodis, N. E., \& North, J. S. (2017). The effects of anxiety and situation-specific context on perceptual-motor skill: A multi-level investigation.

Ryu, D., Abernethy, B., Mann, D. L., Poolton, J. M., \& Gorman, A. D. (2013). The role of central and peripheral vision in expert decision making. Perception, 42(6), 591-607.

Salmela, J. H., \& Fiorito, P. (1979). Visual cues in ice hockey goaltending. Canadian Journal of Applied Sport Sciences = Journal Canadien Des Sciences Appliquées Au Sport, 4(1), $56-59$.

Savelsbergh, G. J. P., Williams, A. M., van der Kamp, J., \& Ward, P. (2002). Visual search, anticipation and expertise in soccer goalkeepers. Journal of Sports Sciences, 20(3), 279287.

Sebanz, N., \& Shiffrar, M. (2009). Detecting deception in a bluffing body: The role of expertise. Psychonomic Bulletin \& Review, 16(1), 170-175. 
Shim, J., Carlton, L. G., \& Kwon, Y. (2006). Perception of kinematic characteristics of tennis strokes for anticipating stroke type and direction. Research Quarterly for Exercise and Sport, 77(3), 326-339.

Singer, R. N. (2000). Performance and human factors: Considerations about cognition and attention for self-paced and externally-paced events. Ergonomics, 43(10), 1661-1680.

Smeeton, N., \& Williams, A. (2012). The role of movement exaggeration in the anticipation of deceptive soccer penalty kicks. British Journal of Psychology, 103(4), 539-555.

Tenenbaum, G., Levy-Kolker, N., Sade, S., Liebermann, D. G., \& Lidor, R. (1996). Anticipation and confidence of decisions related to skilled performance. International Journal of Sport Psychology, 27, 293-307.

Triolet, C., Benguigui, N., Le Runigo, C., \& Williams, A. M. (2013). Quantifying the nature of anticipation in professional tennis. Journal of Sports Sciences, 31(8), 820.

Van der Kamp, J., Rivas, F., Van Doorn, H., \& Savelsbergh, G. (2008). Ventral and dorsal system contributions to visual anticipation in fast ball sports. International Journal of Sport Psychology, 39(2), 100-130.

Vaeyens, R., Lenoir, M., Williams, A. M., Mazyn, L., \& Philippaerts, R. M. (2007). The effects of task constraints on visual search behavior and decision-making skill in youth soccer players. Journal of Sport \& Exercise Psychology, 29(2), 147.

Ward, P., Ericsson, K. A., \& Williams, A. M. (2013). Complex perceptual-cognitive expertise in a simulated task environment. Journal of Cognitive Engineering and Decision Making, 7(3), 231-254. 
Williams, A. M., \& Burwitz, L. (1993). Advance cue utilization in soccer. Science and Football II, 239-244.

Williams, A. M., \& Davids, K. (1998). Visual search strategy, selective attention, and expertise in soccer. Research Quarterly for Exercise and Sport, 69(2), 111.

Williams, A. M., \& Ericsson, K. A. (2005). Perceptual-cognitive expertise in sport: Some considerations when applying the expert performance approach. Human Movement Science, 24, 283-307.

Williams, A. M., Davids, K., \& Williams, J. G. P. (1999). Visual perception and action in sport. New York: E \& FN Spon.

Williams, A. M., North, J. S., \& Hope, E. R. (2012). Identifying the mechanisms underpinning recognition of structured sequences of action. Quarterly Journal of Experimental Psychology, 65(10), 1975-1992. 


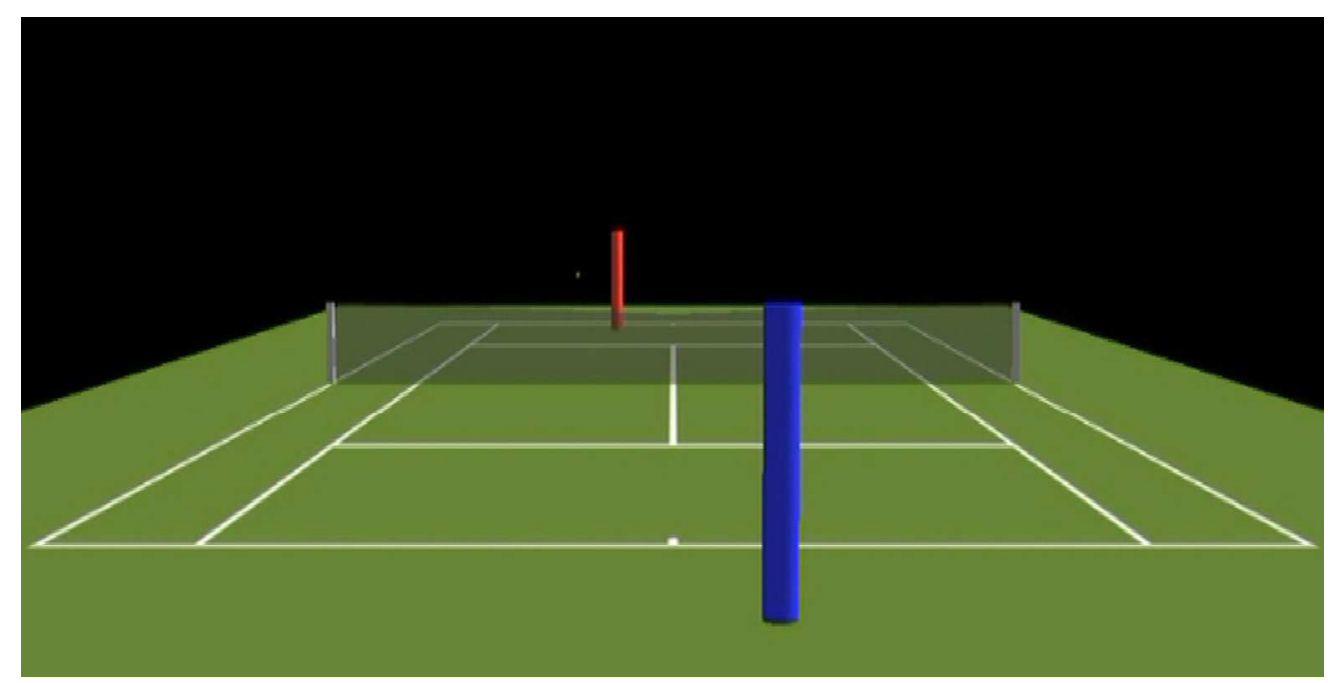

Figure 1. The animated display, created from player movement and ball trajectory data of real matches played by professional players.

$$
311 \times 158 \mathrm{~mm}(96 \times 96 \mathrm{DPI})
$$


Figure 2. Representation of the number of shots played in each Sequence Length condition prior to the same shot occluded at the opponent's racket-ball contact (Exp. 1).

$213 \times 70 \mathrm{~mm}(96 \times 96 \mathrm{DPI})$ 

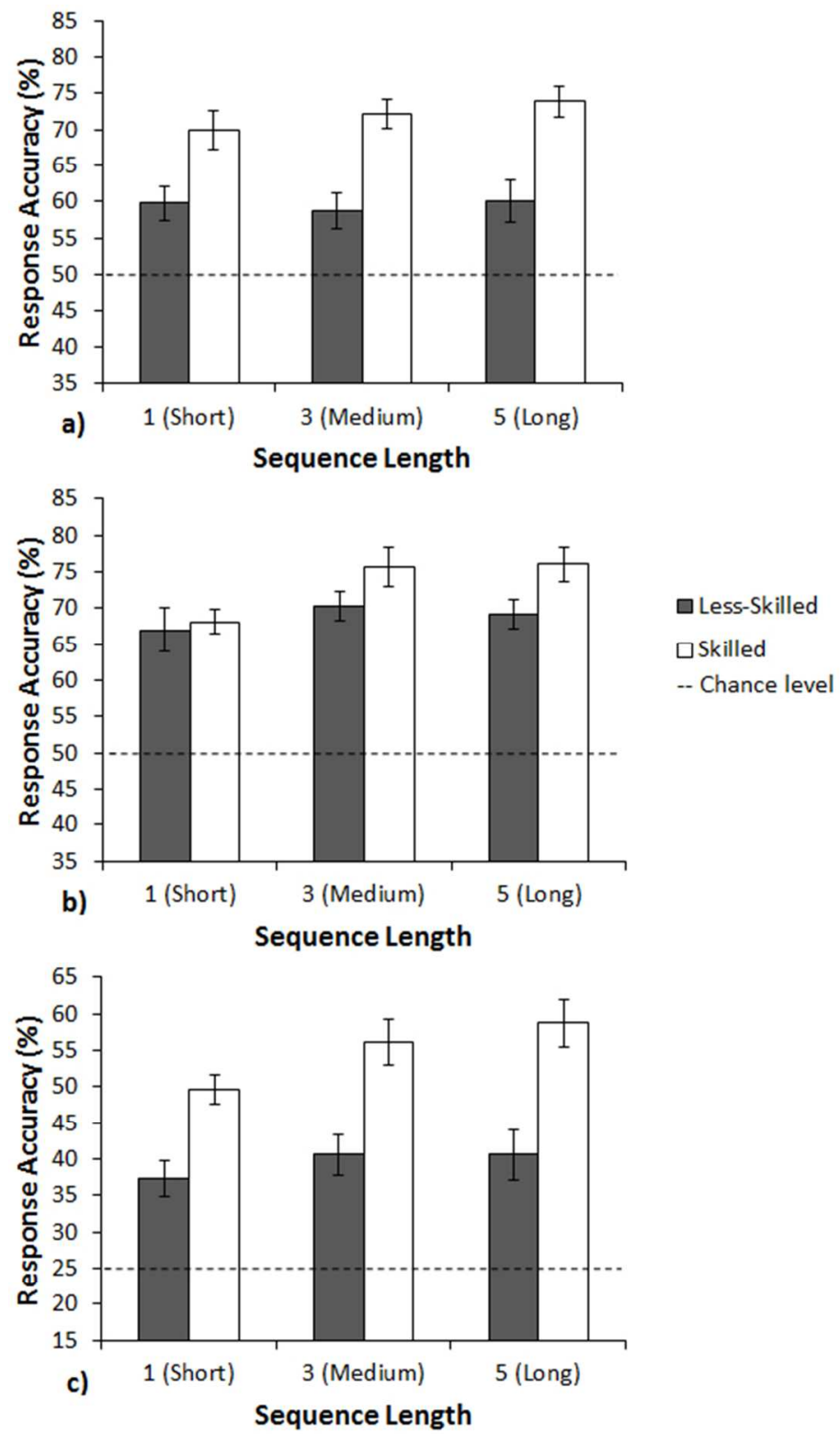

Figure 3. Mean (SE) depth (a), direction (b), and combined (c) response accuracy scores relative to sequence length of skilled (white bars) and less-skilled (grey bars) participants (Exp. 1). Dotted lines represent chance levels.

$140 \times 234 \mathrm{~mm}(96 \times 96 \mathrm{DPI})$ 

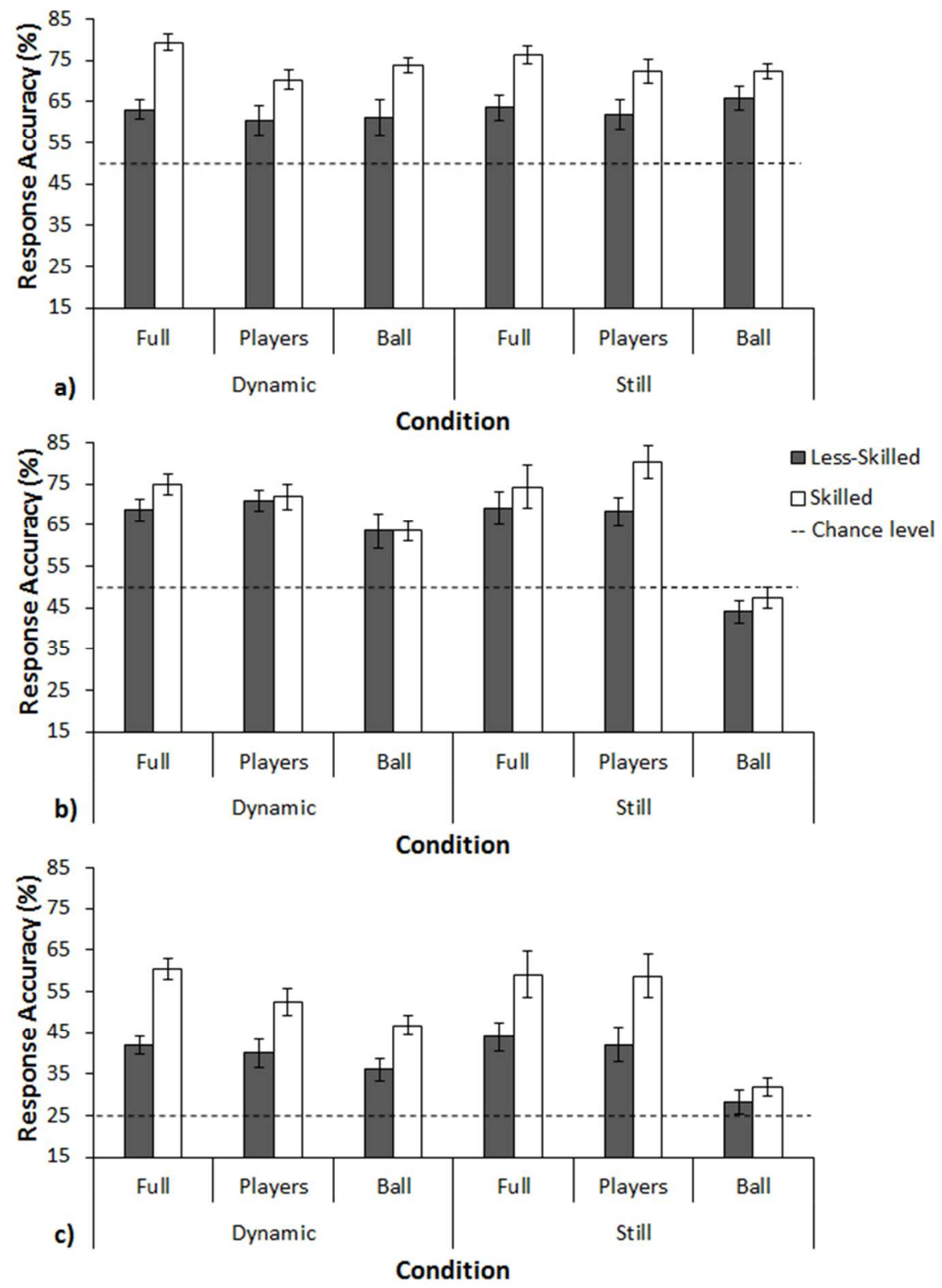

Figure 4. Mean (SE) depth (a), direction (b), and combined (c) response accuracy scores in the six display and presentation conditions of skilled (white bars) and less-skilled (grey bars) participants (Exp. 2). Dotted lines represent chance levels.

$$
166 \times 225 \mathrm{~mm} \text { (96 x } 96 \text { DPI) }
$$


Figure 5. Solution probabilities of skilled and less-skilled participants in a) Dynamic Full, b) Dynamic Players, c) Dynamic Ball, d) Still Full, e) Still Players, and f) Still Ball conditions (Exp. 2). Combined response accuracy values have been rescaled between zero and one.

\section{$387 \times 326 \mathrm{~mm}(96 \times 96 \mathrm{DPI})$}


1

2

3

4

5

6

7

8

9

10

11

12

13

14

15

16

17

18

19

20

21

22

23

24

25

26

27

28

29

30

31

32

33

34

35

36

37

38

39

40

41

42

43

44

45

46

47

48

49

50

51

52

53

54

55

56

57

58

59

60

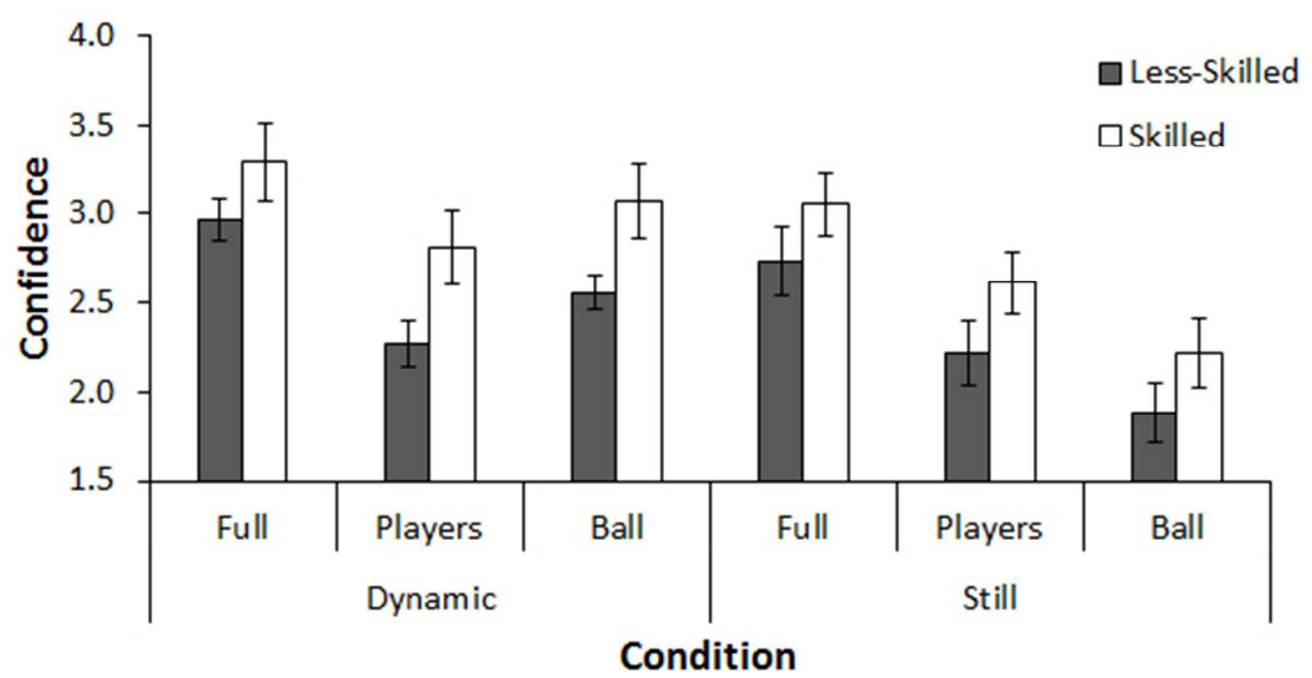

Figure 6. Mean (SE) confidence ratings in the six display and presentation conditions of skilled (white bars) and less-skilled (grey bars) participants (Exp. 2).

$149 \times 77 \mathrm{~mm}(96 \times 96 \mathrm{DPI})$ 